ARTICLE

\title{
Functionalization mediates heat transport in graphene nanoflakes
}

\author{
Haoxue Han ${ }^{1, \star}$, Yong Zhang ${ }^{2,3, \star}$, Nan Wang ${ }^{3, \star}$, Majid Kabiri Samani ${ }^{3}$, Yuxiang $\mathrm{Ni}^{4}$, Zainelabideen Y. Mijbil ${ }^{5,6}$, \\ Michael Edwards ${ }^{3}$, Shiyun Xiong ${ }^{7}$, Kimmo Sääskilahti ${ }^{8}$, Murali Murugesan $^{3}$, Yifeng Fu ${ }^{3,9}$, Lilei Ye ${ }^{9}$, \\ Hatef Sadeghi ${ }^{5}$, Steven Bailey ${ }^{5}$, Yuriy A. Kosevich ${ }^{1,10}$, Colin J. Lambert ${ }^{5}$, Johan Liu $^{2,3}$ \& Sebastian Volz
}

The high thermal conductivity of graphene and few-layer graphene undergoes severe degradations through contact with the substrate. Here we show experimentally that the thermal management of a micro heater is substantially improved by introducing alternative heat-escaping channels into a graphene-based film bonded to functionalized graphene oxide through amino-silane molecules. Using a resistance temperature probe for in situ monitoring we demonstrate that the hotspot temperature was lowered by $\sim 28^{\circ} \mathrm{C}$ for a chip operating at $1,300 \mathrm{Wcm}^{-2}$. Thermal resistance probed by pulsed photothermal reflectance measurements demonstrated an improved thermal coupling due to functionalization on the graphene-graphene oxide interface. Three functionalization molecules manifest distinct interfacial thermal transport behaviour, corroborating our atomistic calculations in unveiling the role of molecular chain length and functional groups. Molecular dynamics simulations reveal that the functionalization constrains the cross-plane phonon scattering, which in turn enhances in-plane heat conduction of the bonded graphene film by recovering the long flexural phonon lifetime.

\footnotetext{
${ }^{1}$ Laboratoire EM2C, CNRS, CentraleSupélec, Université Paris-Saclay, Grande Voie des Vignes, 92295 Châtenay-Malabry, France. ${ }^{2}$ SMIT Center, School of Automation and Mechanical Engineering and Institute of NanomicroEnergy, Shanghai University, 20 Chengzhong Road, Shanghai 201800, China.

3 Electronics Materials and Systems Laboratory, Department of Microtechnology and Nanoscience, Chalmers University of Technology, Kemivägen 9 , SE-412 96 Gothenburg, Sweden. ${ }^{4}$ Department of Mechanical Engineering, University of Minnesota, 111 Church Street SE, Minneapolis, Minnesota 55455, USA. ${ }^{5}$ Quantum Technology Center, Physics Department, Lancaster University, Lancaster LA1 4YB, UK. ${ }^{6}$ Science Department, Veterinary Medicine College, AIQasim Green University, Babylon, Iraq. ${ }^{7}$ Max Planck Institute for Polymer Research, Ackermannweg 10, D-55128 Mainz, Germany. ${ }^{8}$ Department of Biomedical Engineering and Computational Science, Aalto University, FI-00076 Aalto, Finland. ${ }^{9}$ SHT Smart High Tech AB, Ascherbergsgatan 46 , SE-411 33 Gothenburg, Sweden. ${ }^{10}$ Department of Polymers and Composite Materials, Semenov Institute of Chemical Physics, Russian Academy of Sciences, Kosygin Street 4, 119991 Moscow, Russia. * These authors contributed equally to this work. Correspondence and requests for materials should be addressed to C.J.L. (email: c.lambert@lancaster.ac.uk) or to J.L. (email: johan.liu@chalmers.se) or to S.V. (email: sebastian.volz@ecp.fr).
} 
A nisotropic properties of two-dimensional (2D) layered materials make them promising in the application of next-generation electronic devices, among which graphene and few-layer graphene (FLG) have been most intensively studied for thermal management, due to their extraordinarily high in-plane thermal conductivity $(\kappa)^{1-5}$. For instance, Yan et $a l^{6}{ }^{6}$ reported that the maximum hotspot temperature can be lowered by $\sim 20^{\circ} \mathrm{C}$ in transistors operating at $\sim 13 \mathrm{~W} \mathrm{~mm}^{-1}$ using FLG as a heat spreader for a gallium nitride $(\mathrm{GaN})$ transistor. Gao et al..$^{7}$ reported that the maximum hotspot temperature decreased from 121 to $108^{\circ} \mathrm{C}\left(\Delta T=13^{\circ} \mathrm{C}\right)$ for a heat flux of $430 \mathrm{~W} \mathrm{~cm}^{-2}$ after the introduction of a single-layer graphene heat spreader. Moreover, the simulations of graphene heat spreaders were also reported for silicon-on-insulator integrated circuits ${ }^{8}$ and three-dimensional (3D) integrated circuits 9 . The thermal conductivity of a graphene laminate film supported on substrate was also investigated and found to remain rather large ${ }^{10}$. However, in most practical applications, graphene/FLG will be supported by and integrated with insulators, both in electronic circuitry and heat-spreader applications ${ }^{11}$. Therefore, thermal energy flow will be limited both by the in-plane thermal conductivity $(\kappa)$ of the supported graphene/FLG and by the thermal boundary resistance $(R)$ at the graphene/FLG-substrate interface ${ }^{12}$.

Owing to their exceedingly large surface-to-volume ratio, the properties of $2 \mathrm{D}$ layered materials are very sensitive to the interactions with external bodies. Indeed, when supported on an amorphous substrate, $\kappa$ of suspended graphene decreased by almost one order of magnitude, from $\sim 4,000$ (ref. 13) to $\sim 600 \mathrm{~W} \mathrm{~m}^{-1} \mathrm{~K}^{-1}$ (ref. 14). Such a striking discrepancy in $\kappa$ significantly limits the thermal performance of graphene/FLG in real applications. It is reported that the different behaviours are due to the strong scattering of the important heat-carrying flexural acoustic (ZA) modes ${ }^{15}$ to the substrate ${ }^{16}$. More specifically, it was identified that the phonon relaxation times of graphene ZA modes are suppressed when supported on a $\mathrm{SiO}_{2}$ substrate. These studies have improved our fundamental understanding in the physics behind the problem, and it was suggested that making rational choice of the substrate material $^{14,17}$ and modulating its coupling to graphene ${ }^{12}$ may be useful to improve $\kappa$ of the supported graphene/FLG.

The thermal boundary resistance $(R)$ of a graphene/FLGsubstrate interface is another limiting factor to their thermal performance in devices. Covalent functionalization has been proved to efficiently promote heat transfer between interfaces by introducing additional thermal pathways through the functionalizing molecules ${ }^{18-33}$. For example, self-assembled monolayers (SAMs) were used to functionalize metallic surfaces to enhance heat transport across metal-water ${ }^{20,27}$, metal-gas ${ }^{28}$, metalsemiconductor ${ }^{21}$ and metal-polymer ${ }^{31}$ interfaces. Functionalization was used in graphene and carbon nanotube nanocomposites to mitigate the high thermal boundary resistance between the graphene/carbon nanotube fillers and the polymer matrices ${ }^{18,25,26,30}$. Functionalized molecules also assist to align and densely pack multilayer graphene sheets and reduce the interlayer thermal resistance of graphene ${ }^{25}$. Recently, it was shown that plasmafunctionalized graphene raised the cross-plane thermal conductance between aluminium and its substrate by a factor of two ${ }^{19}$. Nevertheless, the functionalization-introduced point defects will further decrease $\kappa$ of the supported graphene/FLG, as they introduce phonon-scattering centres ${ }^{25,32,33}$. To correct this drawback, a robust solution that maintains the high thermal conductivity of graphene/ FLG when supported, while effectively reducing the interface thermal resistance is needed.

Here we demonstrate that thermal management of a micro heater is considerably improved via introducing alternative heat-spreading channels implemented with graphene-based film (GBF) bonded to functionalized graphene oxide (FGO) through amino-silane molecules. We probed interface thermal resistance by photothermal reflectance measurements to demonstrate an improved thermal coupling due to functionalization on the graphene-graphene oxide interface and the graphene oxide-silica interface. Molecular dynamics simulations and $a b$ initio calculations reveal that the functionalization constrains the cross-plane scattering of low-frequency phonons, which in turn enhances in-plane heat conduction of the bonded graphene film by recovering the long flexural phonon lifetime. Our results provide evidence that a graphene film deposited on a FGO substrate provides a very attractive platform for thermal management applications.

\section{Results}

Graphene-based film and graphene oxide and device. A GBF bonded to the FGO substrate through silane molecules is shown in Fig. 1a,b. To synthesize GBF and FGO experimentally (Fig. 1c), we first prepared a graphene oxide (GO) dispersion (see Experimental method). The FGO was obtained by functionalizing GO with a silane-based chemistry suitable for reactive oxideforming surfaces including the basal plane of $\mathrm{GO}$ and $\mathrm{SiO}_{2}$. 3-Amino-propyltriethoxysilane (APTES) has three - $\mathrm{Si}-\mathrm{O}$ - groups and one $-\mathrm{NH}_{2}$ end, as shown in Fig. 1a. Owing to the simple chemistry and unique multifunctional nature of APTES, it can easily bind two different substrates. In our case, the $-\mathrm{Si}-\mathrm{O}$ end of APTES binds to the GO substrate. $-\mathrm{Si}-\mathrm{OC}_{2} \mathrm{H}_{5}$ groups of APTES hydrolyse in water and form crosslink bonds with each other. The crosslinked $\mathrm{Si}-\mathrm{O}$ structure acts as a strong bonding layer between the substrate and GBFs. On the other hand, the $-\mathrm{NH}_{2}$ end of APTES binds onto carboxyl groups on the functionalized graphene film. The FGO layer has a thickness of $\sim 5 \mathrm{~nm}$. The graphene film was fabricated from chemically reduced GO and can recover relatively high in-plane thermal conductivity after thermal annealing ${ }^{34}$. The graphene film was then spin-coated ${ }^{35}$ with the FGO and the resulting bundle was transferred to a thermal evaluation device ${ }^{7}$, resulting in the formation of molecular bridges between the graphene surface and the device's $\mathrm{SiO}_{2}$ substrate. The thermal evaluation device was integrated with micro Pt-based heating resistors as the hotspot and temperature sensors ${ }^{7}$, as shown in Fig. 1d, acting as a simulation platform of an electronic component to demonstrate the heat-spreading capability of the supported graphene film.

In situ temperature measurement with resistance thermometry. A direct current $I$ was input into the circuit by applying an outer voltage $V$ in Fig. 1e, and hence the generated power is calculated as $P=V \times I$. Since the lateral dimension of the hotspot $\left(A=400 \times 400 \mu \mathrm{m}^{2}\right)$ is much larger than its thickness $(260 \mathrm{~nm})$, most of the heat is dissipated through the lateral direction of the hotspot. Hence the heat flux is defined as $Q=P / A$, and the direction is parallel to the substrate. The calibration relationship between the resistance $R(\Omega)$ and the temperature $T\left({ }^{\circ} \mathrm{C}\right)$ of the thermal evaluation chip is $R(T)=0.21 T+112$. The temperature measurement uncertainty is $\epsilon= \pm 0.5^{\circ} \mathrm{C}$. Figure le shows the temperature measured in situ at the hotspot and compares the thermal performance of the graphene film with and without the functionalization. With a constant heat flux of $1,300 \pm 2.3 \mathrm{~W} \mathrm{~cm}^{-2}$ at the $\mathrm{Pt}$ chip, the hotspot temperature decreased from $135 \pm 1.2$ to $118 \pm 1.1^{\circ} \mathrm{C}\left(\Delta T=17 \pm 2.3^{\circ} \mathrm{C}\right)$ with a GBF deposited on non-functionalized GO compared with the case of a bare chip. Such a remarkable temperature decrease is far beyond the measurement uncertainty $\Delta T \gg \epsilon$ (see Supplementary Fig. 1 and Supplementary Note 1 for an uncertainty analysis). 
a
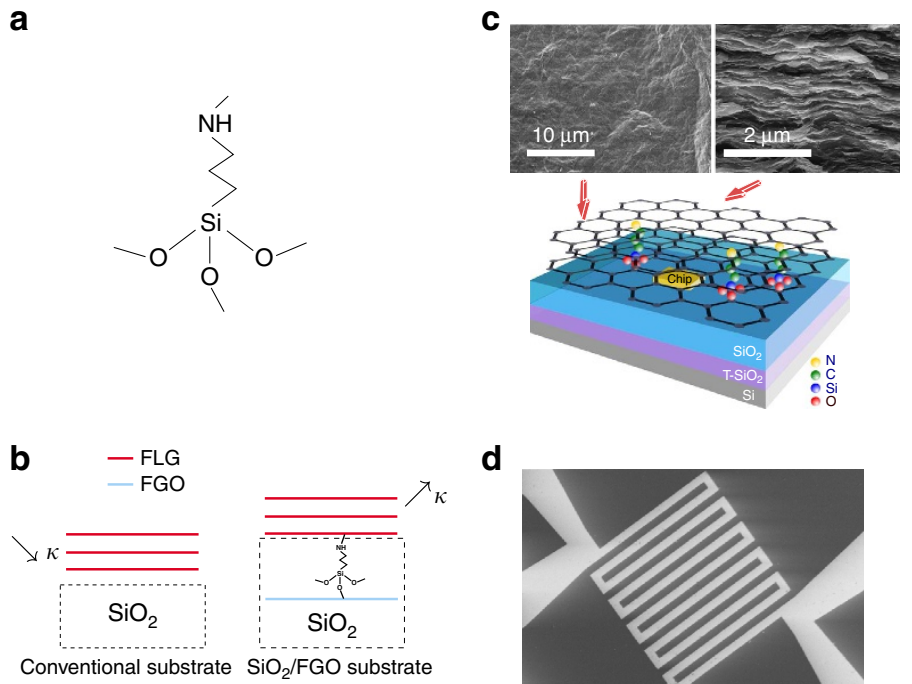

d

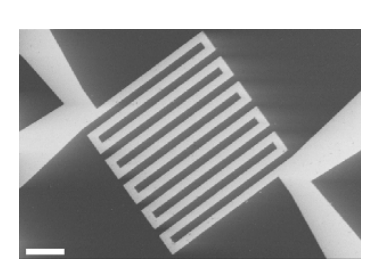

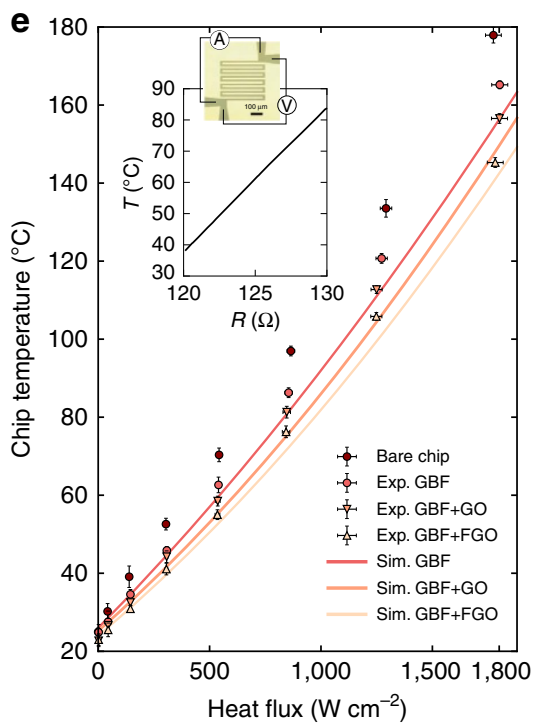

Figure 1 | Graphene-based film on FGO as heat spreader for hotspot. (a) Sketch of the chemical bonds of the silane molecule. (b) Schematic of a graphene film on different supports. Left: conventional silica substrate. Right: the proposed silica/FGO substrate. (c) Schematic of the measurement set-up. The chip is embedded in the $\mathrm{SiO}_{2}$ substrate. $\mathrm{T}-\mathrm{SiO}_{2}$ stands for thermally grown $\mathrm{SiO}_{2}$. Scanning electron microscopy (SEM) image of the in-plane and the cross-section of the GBF. (d) SEM image of the chip as the hotspot. Scale bar, $100 \mu \mathrm{m}$. (e) Measured (filled markers) and finite-element-simulated (lines) chip temperatures versus the in-plane heat fluxes dissipated in a bare hotspot (rectangles), a hotspot covered by a GBF (circles), a chip covered by a GBF with non-functionalized GO (up triangle) and a chip covered by a GBF with APTES FGO (down triangle). 'Exp.' and 'Sim.' stand for experiments and simulations, respectively. Inset: calibration relationship between the resistance $R(\Omega)$ and the temperature $T\left({ }^{\circ} \mathrm{C}\right)$ of the thermal evaluation chip. Scale bar on the chip, $100 \mu \mathrm{m}$. The error bars correspond to the s.d.'s from the measurements on five samples.

Furthermore, with the same heat flux input, the hotspot temperature further decreased from $118 \pm 1.1$ to $107 \pm 0.8^{\circ} \mathrm{C}$ $\left(\Delta T=11 \pm 1.9^{\circ} \mathrm{C}\right)$ thanks to the presence of the APTES functionalization. The heat-spreading performance is thus enhanced by $\sim 57 \%$ via the functionalization. We have implemented a finite-element model (Supplementary Figs 2-4, Supplementary Table 1 and Supplementary Note 2) of the heat-spreading device by taking the results of atomistic simulation as input parameters. As shown in Fig. 1e, the heat-spreading performance of the equivalent macroscopic finite-element model agrees reasonably well with the one measured by experiments.

Thermal resistance measurement with photothermal reflectance. To further confirm the enhanced heat spreading assisted by molecular functionalization, we measured the interface thermal resistance by using the pulsed photothermal reflectance (PPR) method ${ }^{36,37}$. To enhance heat absorption, a gold layer was evaporated on the surface of the GO and FGO layers after drop coating. The sample was first excited by a Nd:YAG laser pulse. This caused a fast rise in the surface temperature followed by a relaxation. The change of surface temperature was monitored by a probe laser, which reflects off from the samples' surface. Since the relaxation time is governed by the thermal properties of the underlying layers and interfacial thermal resistance between the layers, by obtaining the temperature profile one can extract the thermal properties of the layers and interface thermal resistance between the layers through a heat conduction model. Four sets of samples were fabricated, as shown in Fig. 2 and the thermal resistance $R_{1}$ between the $\mathrm{Au}-\mathrm{Cr}$ film and the (functionalized) GO layer, and the resistance $R_{2}$ between (functionalized) GO layer and $\mathrm{GBF}$ or $\mathrm{SiO}_{2}$ were measured. The experimental set-up and the procedure of thermal resistance extraction by fitting the photothermal response to the model (Supplementary Figs 5 and 6 and Supplementary Note 3). The normalized surface temperatures of the four sample sets are
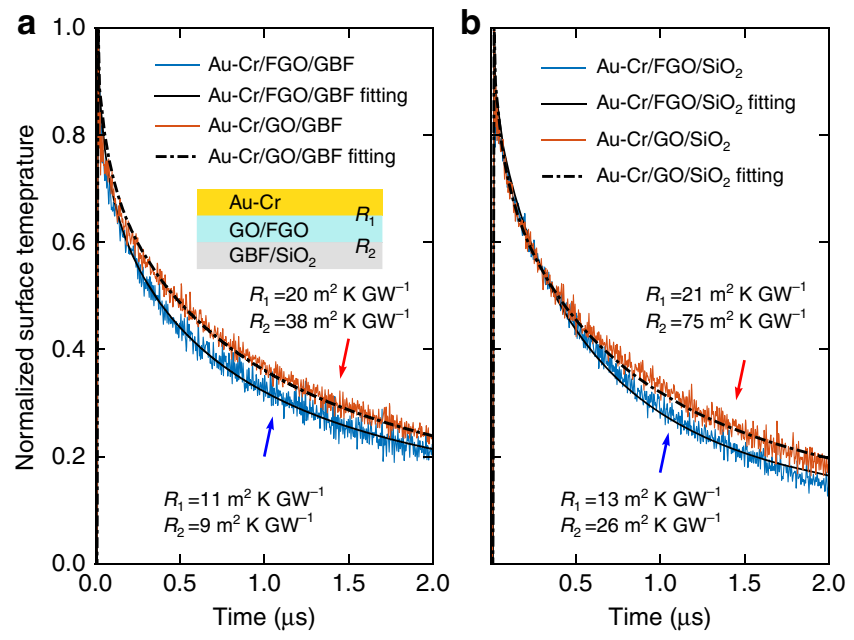

Figure 2 | Normalized surface temperature of PPR measurements of the thermal interface resistance. (a) $\mathrm{Au}-\mathrm{Cr} / \mathrm{FGO} / \mathrm{GBF}$ and $\mathrm{Au}-\mathrm{Cr} / \mathrm{GO} / \mathrm{GBF}$ samples and (b) $\mathrm{Au}-\mathrm{Cr} / \mathrm{FGO} / \mathrm{SiO}_{2}$ and $\mathrm{Au}-\mathrm{Cr} / \mathrm{GO} / \mathrm{SiO}_{2}$ samples. Inset: sample geometry for the PPR measurement. $R_{1}$ and $R_{2}$ refer to the thermal interface resistance between the Au-Cr film and the (APTES functionalized) $\mathrm{GO}$ layer, and that between (functionalized) $\mathrm{GO}$ layer and $\mathrm{GBF}$ or $\mathrm{SiO}_{2}$. The thermal resistances are also reported in Table 1.

shown in Fig. 2 and the extracted thermal resistances are reported in Table 1. A fourfold reduction was achieved in the thermal resistance between $\mathrm{GO}$ and $\mathrm{GBF}$ from $3.8 \times 10^{-8}$ to $0.9 \times 10^{-8} \mathrm{~m}^{2} \mathrm{KW}^{-1}$. On the $\mathrm{GO}-\mathrm{SiO}_{2}$ interface, the functionalization remarkably reduced the thermal resistance by a factor of almost three, from $7.5 \times 10^{-8}$ to $2.6 \times 10^{-8} \mathrm{~m}^{2} \mathrm{KW}^{-1}$. We also observed a better thermal coupling on the metal-dielectric interface between $\mathrm{Au}-\mathrm{Cr}$ and GO due to the surface chemistry. 


\begin{tabular}{|c|c|c|}
\hline Interface & $R_{1}\left(\mathrm{~m}^{2} K \mathrm{~W}^{-1}\right)$ & $R_{2}\left(m^{2} K W^{-1}\right)$ \\
\hline $\mathrm{Au}-\mathrm{Cr} / \mathrm{GO} / \mathrm{GBF}$ & $2.0 \mathrm{E}-8$ & $3.8 \mathrm{E}-8$ \\
\hline $\mathrm{Au}-\mathrm{Cr} / \mathrm{FGO} / \mathrm{GBF}$ & $1.1 \mathrm{E}-8$ & $0.9 \mathrm{E}-8$ \\
\hline $\mathrm{Au}-\mathrm{Cr} / \mathrm{GO} / \mathrm{SiO}_{2}$ & $2.1 \mathrm{E}-8$ & $7.5 \mathrm{E}-8$ \\
\hline $\mathrm{Au}-\mathrm{Cr} / \mathrm{FGO} / \mathrm{SiO}_{2}$ & $1.3 \mathrm{E}-8$ & $2.6 \mathrm{E}-8$ \\
\hline
\end{tabular}
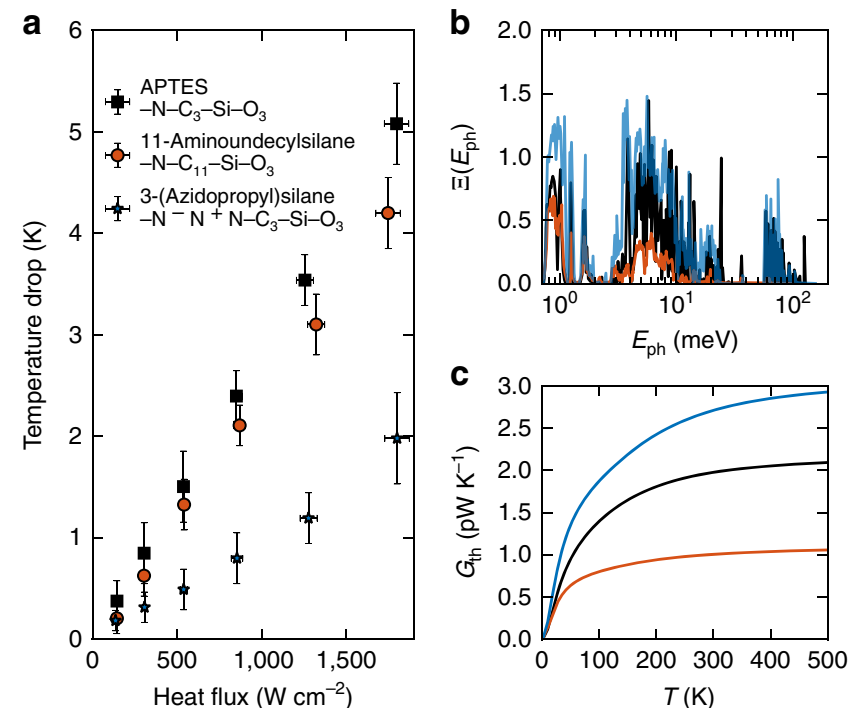

Figure 3 | Heat-spreading performance of GBF on FGO

with different functional agents. The molecules are APTES,

11-Aminoundecyltriethoxysilane and 3-(Azidopropyl)triethoxysilane.

(a) Measured temperature drop of heat spreaders with different functionalization molecules compared with that with non-functionalized GO. The error bars correspond to the s.d.'s of measurements on five samples for each molecule type. (b) Phonon transmission function $\Xi\left(E_{\mathrm{ph}}\right)$ for three different types of molecules used in the experiment. (c) Phonon thermal conductance through the molecules as a function of temperature. The colour code of the data curves in $\mathbf{b}$ and $\mathbf{c}$ is the same as in $\mathbf{a}$.

Heat spreading through different functional molecules. To gain a deeper insight into the impact of molecular structure on the thermal transport along the molecules, we used APTES, 11-Aminoundecyltriethoxysilane and 3-(Azidopropyl)triethoxysilane as different functional agent on the GO to evaluate their heat-spreading performance on the same thermal test platform. The same concentration of $0.1492 \mathrm{~mol} \mathrm{~kg}^{-1}$ was used for all three types of molecules. Figure 3 shows the temperature reduction of the hotspot covered by GBFs with FGO using three different molecules. The results show that the heat spreader of GO functionalized with APTES has the best cooling performance. To properly understand this difference, an exploration of the internal vibrational properties of the molecule is crucial ${ }^{38}$. We hence investigated how the differences in the phonon transmission impact the interfacial thermal transport. With this aim, we probed the phonon transmission $\Xi\left(E_{\mathrm{ph}}\right)$ by atomistic Green's function to characterize the local heat conduction with and without the presence of the molecule (Supplementary Note 4$)$. $\Xi\left(E_{\mathrm{ph}}\right)$ enables a precise measurement of the atomicscale molecule-graphene heat transport that the conventional models fail to provide. The phonon transmission functions through different molecules and the associated thermal
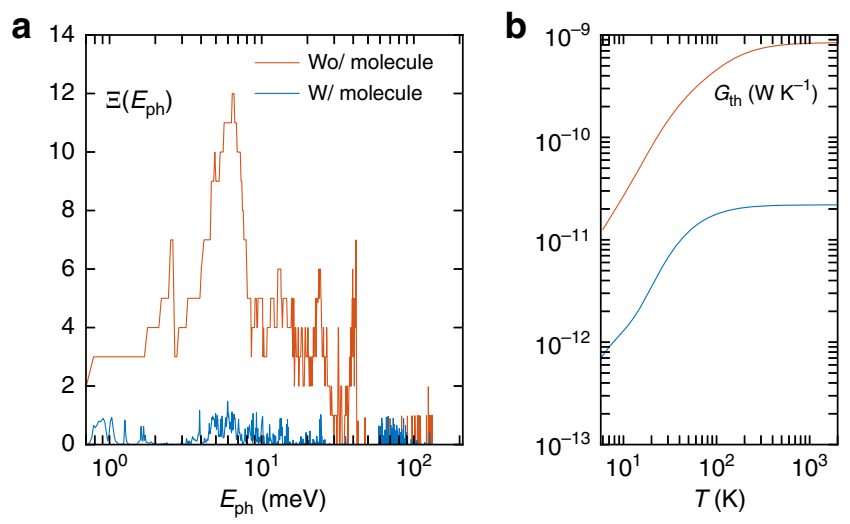

Figure 4 | Phonon transport through the APTES molecule. (a) Phonon transmission $\Xi\left(E_{\mathrm{ph}}\right)$ versus phonon energy $E_{\mathrm{ph}}=\hbar \omega$ (red curve) between two adjacent graphene layers and (blue curve) through the APTES molecule bonding the two graphene layers. 'w/' and 'wo/' stand for with and without, respectively. (b) Thermal conductances $G_{\text {th }}$ contributed by phonons versus temperature for the two cases.

conductances versus temperature are shown in Fig. 3b,c. The phonon transmission at low phonon energies across the 11-Aminoundecyltriethoxysilane molecule is comparable to that across the APTES molecule, whereas at high phonon energies $(>4 \mathrm{meV}$ ), the phonon transmission is considerably suppressed. Such a distinct phonon transport behaviour is determined by the molecule chain length. By comparing the chemical structures, we can see that 11-Aminoundecyltriethoxysilane $\left(-\mathrm{N}-\mathrm{C}_{11}-\mathrm{Si}-\mathrm{O}_{3}\right)$ has the same functional groups as APTES $\left(-\mathrm{N}-\mathrm{C}_{3}-\mathrm{Si}-\mathrm{O}_{3}\right)$ but has a longer carbon backbone. Such a long chain length has a rather weak impact on the low-frequency phonons due to their very long wavelength but can strongly suppress the phonon transport at high frequencies. On the other hand, when comparing the phonon transmission through the junction of APTES and 3-(Azidopropyl)triethoxysilane $\left(-\mathrm{N}^{-} \mathrm{N}^{+} \mathrm{N}-\mathrm{C}_{3}-\mathrm{Si}-\mathrm{O}_{3}\right)$, it is evident to identify a stronger transmission at all frequencies. By comparing the chemical structures in Fig. 3a, we can see that 3-(Azidopropyl)triethoxysilane has the same carbon backbone as APTES but has an azido group instead of an amino group. The azido group has strong interaction with the $s p^{2}$-bonded carbon lattice of graphene to form three-membered heterocycle structures. Given the similar backbone structure, the phonon eigenmodes in the molecule have not been significantly altered, but the transmission is enhanced due to the stronger thermal coupling to the graphene reservoirs. However, the introduction of nitrogen atoms into the $s p^{2}$ carbon structure can strongly scatter phonons by distorting the structure of the graphene sheet, which leads to a poor thermal performance of the heat spreader.

Phonon transport analysis in APTES. We now investigate the detailed vibrational and electronic transport properties of the APTES molecule since it presents the best performance in heat spreading. We probed the phonon transmission $\Xi\left(E_{\mathrm{ph}}\right)$ to characterize the local heat conduction with and without the presence of the APTES molecule. As shown in Fig. 4, the transmission function $\Xi\left(E_{\mathrm{ph}}\right)$ for the two adjacent graphene layers without any molecule displays a clear stepwise structure that provides the number of phonon channels. Low-energy phonons $\left(E_{\mathrm{ph}} \leq 10\right.$ $\mathrm{meV}$ ) dominate heat conduction since the adjacent graphene flakes interact only through weak van der Waals (vdW) forces that inhibit the transmission of high-frequency phonons ${ }^{39}$. When the graphene layers are bridged by a amino-silane molecule, the 
high-frequency phonons act as the major contributors in the heat conductance $G_{\mathrm{ph}}$, creating more phonon channels through the covalent bond vibrations. This is in line with the transmission calculation of Segal et al. ${ }^{40}$ who observed a contribution to heat conduction by the higher-frequency phonons within the molecule coupled to the low-frequency phonons responsible for heat transport in the thermal reservoirs. The oscillations in the transmission spectrum may originate from phonon interferences within the alkane chain ${ }^{41-43}$. Fabry-Pérot-like interference effect occurs in the frequency region of $E_{\mathrm{ph}}=20-100 \mathrm{meV}$, as was previously observed in an alkane SAM interface ${ }^{41}$. Such Fabry-Pérot-like interferences originate from the multiple reflected phonons interfering constructively within the alkane chain, as the local maxima in the transmission (Fig. 4) through the molecule can attain the same intensity of that through pristine graphene films at given frequencies. Although destructive quantum interference was believed unlikely to occur in a linear alkane chain ${ }^{42}$, we observe strong destructive interference patterns in the high-frequency range $(\omega / 2 \pi=40-60 \mathrm{THz})$, which may correspond to two-path destructive phonon interferences ${ }^{43,44}$. We also investigated the interlayer electron transport in the graphene and the effect of the silane intercalation in such a hybrid nanostructure through a ab initio calculation combined with Green's function (Supplementary Fig. 8 and Supplementary Note 5). The main heat carrier in this system is phonon, as the thermal conductance due to the electron contributes to the total thermal conductance by $\sim 4 \%$ at room temperature with functionalization and by $\sim 2 \%$ without molecules.

Thermal conductance and conductivity calculations. To explore the effect of functional APTES molecules on the in-plane thermal conductance of the graphene film, we first perform molecular dynamics simulations to study a nanoscale molecular junction between two stacks of multilayer graphene nanoflakes. For a weak oxidation, the thermal resistance of the graphene film and its oxidized substrate on one hand, and on the other hand its thermal conductivity are very close to those with a non-oxidized graphene support. The dependence of thermal resistance and conductivity of graphene film to the oxidation rate of the GO substrate is reported in Supplementary Fig. 9 and Supplementary Note 6. We consider defect-free and isotopically pure graphene flakes of $10 \times 10 \mathrm{~nm}^{2}$ thermalized at $300 \mathrm{~K}$ (Methods, Supplementary Fig. 10 and Supplementary Note 7). The FGO substrate has a 5-nm thickness as in the experiments. Conventional silica substrate results in a substantial decrease of the basal-plane thermal conductivity of graphene due to the nonconformality of the substrate-graphene interface ${ }^{14}$. To compare, the FGO substrate proposed herein minimize the perturbation of substrate on the morphology of graphene (Fig. 1c), thus maintaining its high thermal conductivity. Periodic boundary conditions were used in the in-plane directions so that the molecular dynamics system corresponds to two thin films connected through silane molecules with the number density $\rho$, defined as the molecule number per graphene unit area.

To illustrate the intriguing role of the functionalizing molecule, the in-plane thermal conductivity $\kappa$ of the film and its interfacial thermal resistance $R$ (Supplementary Fig. 10 and Supplementary Note 7) with the FGO substrate is plotted as a function of the graphene layer number $l_{G}$ in the film in Fig. 5a,b, respectively. First, a supercell containing a single molecule is studied, which corresponds to $\rho=0.081 \mathrm{~nm}^{-2}$. For $l_{\mathrm{G}} \geq 2$, the presence of the APTES molecule results in an unexpected increase both in the graphene film thermal conductivity $\kappa$ and in $R$. An overall decaying trend of the in-plane thermal conductivity $\kappa$ of the

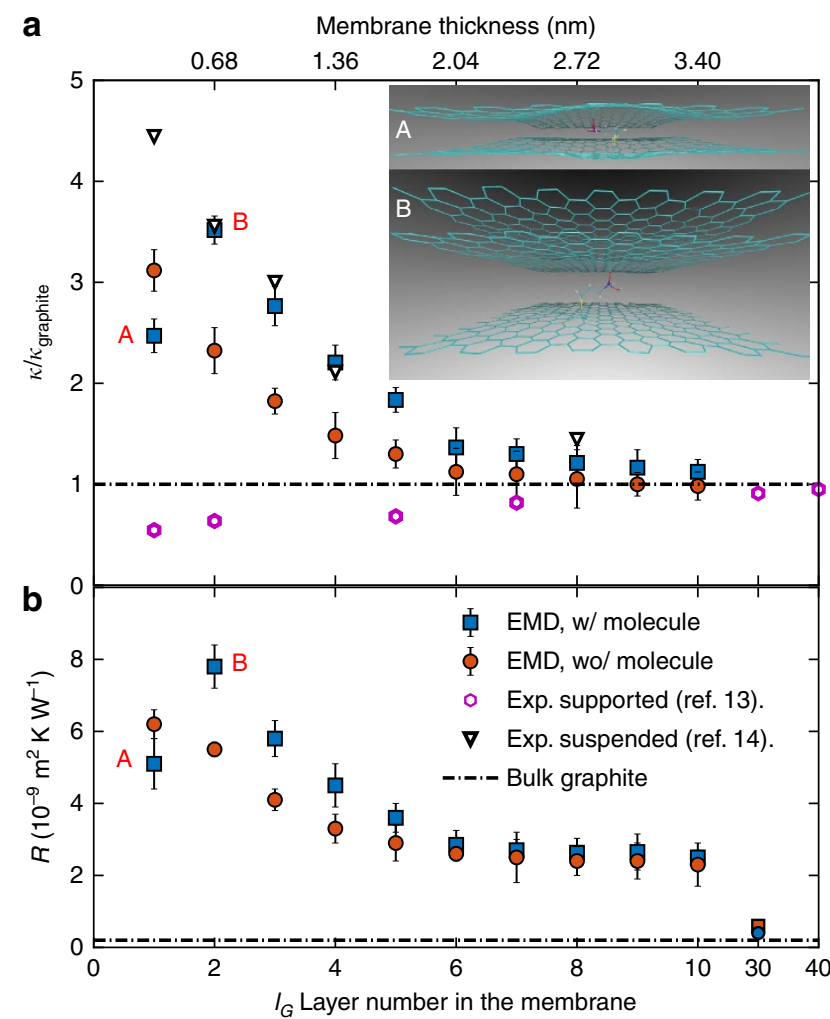

Figure 5 | Thermal resistance and in-plane thermal conductivity of the GBF versus the graphene layer number. (a) Molecular dynamics simulation results of in-plane thermal conductivity $\kappa$ of the graphene film and (b) interfacial thermal resistance $R$ between the FGO substrate and the graphene film versus the graphene layer number $I_{G}$ in the film. The molecule density is $\rho=0.081 \mathrm{~nm}^{-2}$. Cases with (red circles) and without (blue squares) the APTES molecule are compared. 'w/' and 'wo/' stand for with and without, respectively. The values of thermal conductivity are normalized to that of the single-layer graphene. Inset A illustrates a saddlelike surface generated by the APTES molecule for $I_{G}=1$. Inset $B$ shows that the saddle-like curvature disappears for $I_{G} \geq 1$. The measurements of suspended and supported graphene are from refs 13,14, respectively. EMD stand for equilibrium molecular dynamics. 'Exp.' and 'Sim.' stand for experiments and simulations, respectively. The simulated (respectively measured) thermal conductivity is normalized with respect to that of the simulated (respectively measured) graphite to allow a reasonable comparison. The error bar corresponds to the s.d. of the ensemble average on 50 independent trajectories.

graphene film and its resistance $R$ with the substrate versus the layer number $l_{\mathrm{G}}$ is observed until approaching the value of bulk graphite. This is due to the increased cross-plane coupling of the low-energy phonons. A similar decay was found both in experimental measurements of $\kappa$ (ref. 13) of suspended graphene and in simulation-based estimation of $R$ (ref. 45). Unlike silicasupported graphene ${ }^{14}$, the FLG on FGO support recovers the high thermal conductivity and follows the same decaying trend versus $l_{\mathrm{G}}$ as the suspended graphene ${ }^{13}$. Therefore, the proposed FGO substrate better conserves the high thermal conductivity of graphene compared with the silica substrate. Interestingly, for $l_{\mathrm{G}}=1$, the presence of the molecule reduces $\kappa$, which goes against the case where no molecule interconnects the graphene film and the substrate. This breakdown of the thermal conductivity enhancement is due to a saddle-like surface generated around the molecule's chemical bonds of amino and silano groups connecting the graphene, with the bond centre as the saddle point, as shown in the inset A of Fig. 5a. The saddle-like surface 
strongly scatters all phonon modes, thus decreasing $\kappa$ of the graphene film. Such a curved surface was found quite common in defective graphene ${ }^{46,47}$, resulting from the Jahn-Teller effect to lower the energy by geometrical distortion ${ }^{48}$. The thermal conductance of a single silane molecule is determined to be $82 \mathrm{pW} \mathrm{K}^{-1}$ through the molecular footprint (Supplementary Fig. 11 and Supplementary Note 8). Our simulated result is comparable to recent measurements of the thermal conductance of alkane thiols SAM at a silica-gold interface ${ }^{38}$.

It is clearly seen from Fig. $5 \mathrm{a}$ that the critical layer number for the in-plane thermal conductivity switch is $l_{c}=2$. This critical number depends on the level of conformation distortion of the functionalized graphene sheet. Several factors can play a role (Supplementary Figs 12-14 and Supplementary Note 9): (i) molecule density: a diluted molecule distribution creates segregated ripples on the functionalized graphene sheet and even on the sheets further above thus interrupting the phonon mean free path. In this case more graphene sheets are required to recover the flat conformation, that is, $l_{\mathrm{c}}>2$. For a high molecule density, the ripples tend to merge with themselves, recovering the flat surface in an extended area. Such merging hence recovers the interrupted phonon MFP and alleviates the detrimental influence of the separate ripples on the basal-plane thermal conductivity of graphene. In this case the two layers of graphene with functionalization have higher basal-plane thermal conductivity compared with that without functionalization, that is, $l_{c}=2$. (ii) Functional group of the molecule: when the functional agent forms a stronger bond with its substrate than that of the aminosilane molecule, more severe static graphene distortion occurs thus increasing the critical number $l_{c}$. (iii) Substrate of the graphene film: a highly mismatched substrate yields remarkable surface distortion of the graphene sheets in such a way that two layers of graphene are not sufficient to recover the high basal-plane thermal conductivity.

Functionalization effects on phonon lifetime of graphene. We investigate the microscopic origin of the thermal conductivity $\kappa$ enhancement in the graphene film by probing the mode-wise phonon relaxation time (Supplementary Fig. 15 and Supplementary Note 10). The phonon relaxation time $\tau$ measures the temporal response of a perturbed phonon mode to relax back to equilibrium due to the net effect of different phonon-scattering mechanisms. $\tau$ can be defined as ${ }^{49} \partial n / \partial \tau=\left(n-n_{0}\right) / \tau$ where $n$ and $n_{0}$ are the phonon occupation numbers out of and at thermal equilibrium. Under the single-mode-relaxation-time approximation, the thermal conductivity is given by $\kappa=\sum C_{i} v_{i}^{2} \tau_{i}$ where $C_{i}$ and $v_{i}$ are the specific heat per volume unit and the group velocity of the $i$-th phonon mode. The phonon dispersion of the supported graphene film for $\rho=0.081 \mathrm{~nm}^{-2}$ and $l_{\mathrm{G}}=2$, and the extracted relaxation time $\tau$ for all the phonon modes are shown in Fig. 6 . By inserting the APTES molecule, the relaxation time of the acoustic flexural modes $\tau_{\mathrm{ZA}}$ largely increase at low frequencies $(\omega<10 \mathrm{THz})$, whereas the longitudinal and transverse modes undergo a slight decrease in $\tau_{\mathrm{LA}, \mathrm{LO}, \mathrm{TA}, \mathrm{TO}}$. The notable increase in $\tau_{\mathrm{ZA}}$ accounts for the enhancement in $\kappa$ of the graphene film bonded to the substrate since the ZA modes contribute considerably to $\kappa$ as much as $77 \%$ at $300 \mathrm{~K}$ (ref. 15). We attribute the increase in $\tau_{\mathrm{ZA}}$ to the weakened coupling between the graphene film and the substrate, which is reflected as the increased thermal resistance $R$ for $\rho=0.081 \mathrm{~nm}^{-2}$ and $l_{\mathrm{G}} \geq 2$, as is shown in Fig. 5b. An approximate expression from the perturbation theory for the relaxation time due to phonon leakage towards the contact interface yields $\tau^{-1} \propto g(\omega) K^{2} / \omega^{2}$, where $g(\omega)$ depends on the phonon density of states and $K$ is the average vdW coupling constant between the graphene film and its
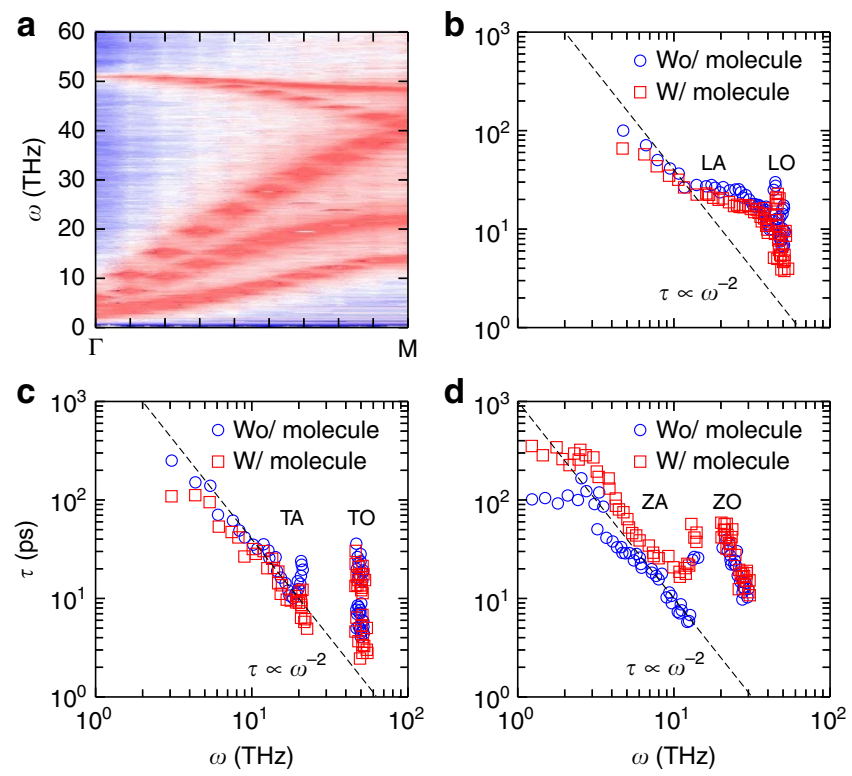

Figure 6 | Phonon dispersion and lifetimes of the graphene film.

(a) Phonon dispersion of the graphene film bonded to the APTES-FGO substrate from molecular dynamics simulations, for $\rho=0.081 \mathrm{~nm}^{-2}$ and $I_{\mathrm{G}}=2$. (b-d) Mode-wise phonon relaxation time for longitudinal modes including longitudinal acoustic (LA) and optical (LO) branches, for transverse modes including transverse acoustic (TA) and optical (TO) branches, and for flexural modes including flexural acoustic (ZA) and optical (ZO) branches, respectively. 'W/' and 'wo/' stand for with and without, respectively. For low-frequency phonons, $\tau$ follows the $\omega^{-2}$ scaling rule of the Umklapp processes at low frequency and high-temperature limits.

substrate. A previous calculation ${ }^{16}$ shows that $K_{\mathrm{ZA}}$ largely depends on the substrate morphology. The presence of the molecule strongly decreases the film-substrate coupling $K_{\mathrm{ZA}}$, thus yielding a longer time $\tau_{\mathrm{ZA}}$. This demonstrates that the aminosilane molecules refrain the film-substrate phonon scattering, which in turn enhances in-plane heat conduction in the bonded graphene film. Note that ZA mode becomes 'massive' as shown in Fig. 6a, that is, the ZA branch does not reach all the way to zero frequency but shifts to higher frequencies. The reduced interplane scattering compensates the reduction in the phonon group velocity at Brillouin zone centre, so that the in-plane thermal conductivity increases after all (Supplementary Fig. 16 and Supplementary Note 11).

The cross-plane heat conduction between the top graphene films and the lower FGO layers is governed by the competing effects of (i) the intercalation of the molecules that tends to weaken the interlayer coupling of graphene therefore increasing the cross-plane thermal resistance and (ii) the additional heat channels introduced by the molecules covalently bonding the graphene sheets that tend to facilitate the cross-plane thermal coupling. We investigated the in-plane thermal conductivity $\kappa$ of the graphene film and its thermal resistance $R$ with the functionalized substrate versus the equivalent APTES molecule number density $\rho$ in Fig. 7. At a low molecule density, the molecule population is too small in such a way that the additional thermal phonon transport through the molecules cannot compensate the weakened cross-plane thermal coupling through the interlayer vdW interactions due to the intercalation effect by the molecules (also see Supplementary Fig. 17 and Supplementary Note 12 for a detailed analysis on the $\kappa-\rho$ relation at low molecular densities). Therefore, the net effect is an increase in the 


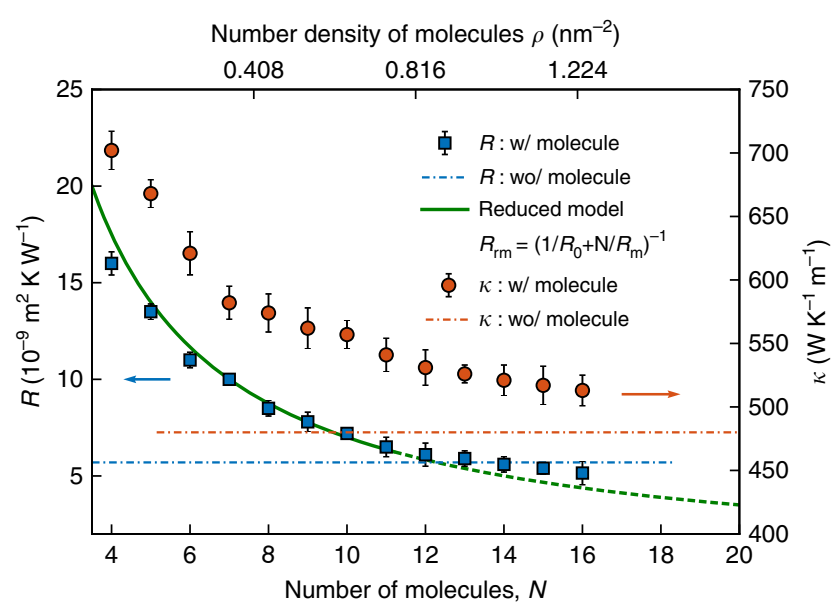

Figure 7 | Thermal resistance and in-plane thermal conductivity of the graphene film versus the molecule density. Thermal conductivity $\kappa$ (red circles) of the graphene film and its thermal resistance $R$ (blue squares) with the APTES functionalized substrate versus the equivalent molecule number density $\rho$. Green line: resistance $R_{\mathrm{rm}}$ predicted by a reduced model of parallel thermal resistors is to compare with the molecular dynamics (MD) results for small $\rho . R_{\mathrm{rm}}=\left(1 / R_{\mathrm{O}}+N / R_{\mathrm{m}}\right)^{-1}$, where $R_{\mathrm{O}}$ is the thermal resistance between two adjacent graphene layers and $R_{\mathrm{m}}$ is the additional resistance induced by a single molecule. The values of $R_{0}$ and $R_{\mathrm{m}}$ are determined in MD simulations: $R_{0}=0.203 \mathrm{~mm}^{2} \mathrm{KW}^{-1}$ and $R_{\mathrm{m}}=0.069 \mathrm{~mm}^{2} \mathrm{KW}^{-1}$. Red and blue dashed lines represent, respectively, $\kappa$ and $R$ without the interconnecting molecule at the film-substrate interface. 'w/' and 'wo/' stand for with and without, respectively. The error bar corresponds to the s.d. of the ensemble average on 50 independent trajectories.

cross-plane thermal resistance between the top graphene films and the lower GO layers compared to the non-functionalization case. In the limit of small number density, we consider the molecules as independent heat conductors connecting the film and the substrate. $R$ agrees well with a reduced model of parallel thermal resistors. Thus, $R$ decreases with the number of molecules. $\kappa$ also decreases with the molecule number since the molecules increase the cross-plane phonon scattering in the graphene film. For larger $\rho$, the interactions among the molecules gain importance and $R$ starts to deviate from the prediction of the parallel-thermal-resistor model, as shown in Fig. 7. The molecule population is large enough so that the additional thermal phonon transport through the molecules becomes very strong and compensates the weakened cross-plane thermal coupling through the interlayer interactions due to the intercalation effect by the molecules. Therefore, the net effect is that the cross-plane thermal resistance between the top graphene films and the lower FGO layers falls lower than that of the case without functionalization in the GO. For large $\rho$, the thermal resistance $R$ is lower than that without molecules. However $\kappa$ remains enhanced by a factor of $\sim 15 \%$ compared with its value for the non-functionalized graphene substrate. The molecule density $\rho$ effectively tunes the thermal conductivity of the supported graphene.

\section{Discussion}

The correlation between the in- and cross-plane thermal transport of 2D materials has been recently studied from both theoretical $^{15,17}$ and experimental ${ }^{13,14,16}$ points of view. Among them, Ghosh et al. ${ }^{13}$ studied the dimensional crossover from a 2D graphene to a $3 \mathrm{D}$ graphite system. The in-plane thermal conductivity decreased with the increase of the layer number in the FLG. Wei et al. ${ }^{17}$ used a 2D material model to show a negative correlation between the in-plane thermal conductivity and the cross-plane interaction. These studies have considered different mechanisms of the correlation, yet no general conclusion is applicable to any quasi-2D system. We show that the functionalization molecules mediate the cross-plane phonon scattering and in turn effectively control the in-plane thermal transport in the graphene-based heat spreader.

Our work tackles the key technological challenge of efficient thermal management in the industry of the next-generation integrated circuit. The excessive heat from the hotspots accelerates the failure rate and slows down the operating speed of microelectronic devices ${ }^{50,51}$. Extremely high power density beyond $1,000 \mathrm{~W} \mathrm{~cm}^{-2}$ can be observed in microprocessors ${ }^{51}$. Coolers are most commonly based on metallic materials due to their relatively high thermal conductivities, for example, $200-400 \mathrm{~W} \mathrm{~m}^{-1} \mathrm{~K}^{-1}$ for $\mathrm{Al}$ and $\mathrm{Cu}$, and the low cost. However, for these technologically important metals, the thermal conductivity of a 100-nm-thick film constitutes only $\sim 20 \%$ of the thermal conductivity of the bulk ${ }^{52,53}$. On the other hand, GBF used in the present work can have an in-plane thermal conductivity as high as $1,600 \mathrm{~W} \mathrm{~m}^{-1} \mathrm{~K}^{-1}$ (ref. 54), which is more than two orders of magnitude higher than the ones of the metal thin films. Such a superior thermal conductivity significantly facilitates the heat conduction when GBFs are used as heat spreaders together with a better cross-plane thermal coupling with FGO. The APTES functionalization yields an additional temperature decrease $\Delta T$ of the hotspot of $11^{\circ} \mathrm{C}$ compared with the case of GBF with non-functionalized GO $\left(\Delta T=17^{\circ} \mathrm{C}\right)$, resulting in a large total temperature decrease of $28^{\circ} \mathrm{C}$ when operating at $1,300 \mathrm{~W} \mathrm{~cm}^{-2}$. In comparison, the highest $\Delta T$ achieved so far at a similar high heat flux of $1,250 \mathrm{~W} \mathrm{~cm}^{-2}$ is $19^{\circ} \mathrm{C}$ by using a miniaturized thermoelectric cooler $^{51}$. The contribution of functionalization to the overall $\Delta T$ is robust especially at higher heat flux beyond $1,000 \mathrm{~W} \mathrm{~cm}^{-2}$, as shown in Fig. 1e. Therefore, we propose a significant packagelevel solution for the thermal management of hotspots in high-power electronics at the micro- and nanometre length scale.

\section{Methods}

Experimental method. (i) Sample synthesis: graphite (Sigma, $4 \mathrm{~g}) ; \mathrm{H}_{2} \mathrm{SO}_{4}(92 \mathrm{ml}$, $98 \%) ; \mathrm{NaNO}_{3}(2 \mathrm{~g})$; and $\mathrm{KMnO}_{4}(12 \mathrm{~g})$ were used to prepare $\mathrm{GO}$ dispersion by following Hummers' method ${ }^{55}$. The obtained GO dispersion was reduced by L-ascorbic acid, and polyvinyl alcohol was also added for better suspension. The GBF was prepared via vacuum filtration with polycarbonate filter paper with a pore size of $3 \mu \mathrm{m}$. The film thickness was controlled by the filtration volume and the graphene concentration in the suspension. After dissolving the filter paper in pure acetone, a freestanding GBF was obtained. The thickness of the GBF was measured as $\sim 20 \mu \mathrm{m}$. Raman spectroscopy data of the GBF before and after the spin-coating with FGO are shown in Supplementary Fig. 18. (ii) Functionalization: GO powder $(20 \mathrm{mg})$ and dicyclohexylcarbodiimide $(5 \mathrm{mg})$ were mixed with APTES $(30 \mathrm{ml})$ by ultrasonication for $2 \mathrm{~h}$ to produce a homogeneous suspension. Then the suspension was heated up to $100^{\circ} \mathrm{C}$ for $3 \mathrm{~h}$, with continuous stirring to realize the functionalization. Fourier transform infrared spectroscopy data provide evidence for the functionalization (Supplementary Fig. 19). (iii) Transfer process: the GBF was first transferred onto a thermal release tape, and then spin-coated ${ }^{35}$ with the FGO layer at 4,000 r.p.m. for 2 min onto the film. The thermal release tape was removed by heating the device.

Simulation set-up. Classical molecular dynamics simulations were performed using LAMMPS ${ }^{56}$. Adaptive intermolecular reactive empirical bond order potential ${ }^{57}$ was used to simulate the graphene's $\mathrm{C}-\mathrm{C}$ interactions. The intramolecular forces are taken into account through the ReaxFF potential ${ }^{58}$, which uses distance-dependent bond-order functions to represent the contributions of chemical bonding to the potential energy. Periodic boundary conditions are applied in the in-plane directions and free boundary condition in the cross-plane direction of the graphene system. First, each supercell was relaxed at the simulation temperature to achieve zero in-plane stress. Then the systems were thermalized by using a Langevin heat bath. The system reached thermal equilibrium after $2 \mathrm{~ns}$ computed in the microcanonical ensemble. Temperature and heat flux were sampled in the microcanonical ensemble in the in the following $1 \mathrm{~ns}$. 
Autocorrelation functions for the resistance and the thermal conductivity were calculated over this latter duration.

Thermal conductance. Thermal conductance $\kappa=\kappa_{\mathrm{e}}+\kappa_{\mathrm{ph}}$, where $\kappa_{\mathrm{ph}}\left(\kappa_{\mathrm{e}}\right)$ is the thermal conductance due to the phonons (electrons). From the phonon transmission $\Xi\left(E_{\mathrm{ph}}\right)$ the thermal conductance due to the phonon could be calculated as $\kappa_{\mathrm{ph}}(T)=\int_{0}^{\infty} \mathrm{d} \omega\left(-\frac{\partial f_{\mathrm{BE}}}{\partial E}\right) \hbar \omega \Xi(\omega) / 2 \pi$, where $\omega$ is the frequency, $T$ refers to the mean temperature of the system, $f_{\mathrm{BE}}$ is the Bose-Einstein phonon statistics, and $\hbar$ represents the reduced Plancks constant. The thermal conductance due to the electrons ${ }^{59}$ could be calculated from the electron transmission coefficient $T_{\mathrm{el}}$ as $\kappa_{\mathrm{e}}(T)=\frac{L_{0} L_{2}-L_{1}^{2}}{h T L_{0}}$, where $L_{n}=\int_{-\infty}^{\infty} \mathrm{d} E\left(E-E_{\mathrm{F}}\right)^{n} T_{\mathrm{el}}(E)\left(-\frac{\partial f_{\mathrm{FD}}}{\partial E}\right)$ in which $f_{\mathrm{FD}}$ is the Fermi-Dirac electron statistics.

\section{References}

1. Yan, Z., Nika, D. L. \& Balandin, A. A. Thermal properties of graphene and few-layer graphene: applications in electronics. IET Circ. Device. Syst. 9, 4-12 (2015).

2. Geim, A. K. \& Novoselov, K. S. The rise of graphene. Nat. Mater. 6, 183-191 (2007).

3. Balandin, A. A. et al. Superior thermal conductivity of single-layer graphene Nano Lett. 8, 902-907 (2008).

4. Balandin, A. A. Thermal properties of graphene and nanostructured carbon materials. Nat. Mater. 10, 569 (2011).

5. Kong, Q. Q. et al. Hierarchical graphenecarbon fiber composite paper as a flexible lateral heat spreader. Adv. Funct. Mater. 24, 4222-4228 (2014).

6. Yan, Z., Liu, G., Khan, J. M. \& Balandin, A. A. Graphene quilts for thermal management of high-power GaN transistors. Nat. Commun. 3, 827 (2012).

7. Gao, Z., Zhang, Y., Fu, Y., Yuen, M. M. \& Liu, J. Thermal chemical vapor deposition grown graphene heat spreader for thermal management of hot spots. Carbon 61, 342-348 (2013).

8. Subrina, S., Kotchetkov, D. \& Balandin, A. A. Heat removal in silicon-oninsulator integrated circuits with graphene lateral heat spreaders. IEEE Electron Device Lett. 30, 1281-1283 (2009).

9. Subrina, S. Modeling based design of graphene heat spreaders and interconnects in 3-D integrated circuits. J. Nanoelectron. Optoelectron. 5, 281-286 (2010).

10. Malekpour, H. et al. Thermal conductivity of graphene laminate. Nano Lett. 14, 5155 (2014).

11. Prasher, R. Graphene spreads the heat. Science 328, 185-186 (2010).

12. Ong, Z. Y. \& Pop, E. Effect of substrate modes on thermal transport in supported graphene. Phys. Rev. B 84, 075471 (2011).

13. Ghosh, S. et al. Dimensional crossover of thermal transport in few-layer graphene. Nat. Mater. 9, 555 (2010).

14. Sadeghi, M. M., Jo, I. \& Shi, L. Phonon-interface scattering in multilayer graphene on an amorphous support. Proc. Natl Acad. Sci. USA 110, 16321 (2013).

15. Lindsay, L., Broido, D. A. \& Mingo, N. Flexual phonons and thermal transport in graphene. Phys. Rev. B 82, 115427 (2010)

16. Seol, J. H. et al. Two-dimensional phonon transport in supported graphene. Science 328, 213 (2010).

17. Wei, Z., Chen, Y. \& Dames, C. Negative correlation between in-plane bonding strength and cross-plane thermal conductivity in a model layered material. Appl. Phys. Lett. 102, 011901 (2013).

18. Luo, T. \& Lloyd, J. R. Enhancement of thermal energy transport across graphene/graphite and polymer interfaces: a molecular dynamics study. Adv. Mater. 22, 2495 (2012).

19. Hopkins, P. E. et al. Manipulating thermal conductance at metal-graphene contacts via chemical functionalization. Nano Lett. 12, 590 (2012).

20. Ge, Z., Cahill, D. G. \& Braun, P. V. Thermal conductance of hydrophilic and hydrophobic interfaces. Phys. Rev. Lett. 96, 186101 (2006).

21. Wang, R. Y., Segalman, R. A. \& Majumdar, A. Room temperature thermal conductance of alkanedithiol self-assembled monolayers. Appl. Phys. Lett. 89, 173113 (2006)

22. Ramanathan, T. et al. Functionalized graphene sheets for polymer nanocomposites. Nat. Nanotechnol. 3, 327-331 (2008).

23. Konatham, D. \& Striolo, A. Thermal boundary resistance at the graphene-oil interface. Appl. Phys. Lett. 95, 163105 (2009).

24. Collins, K. C., Chen, S. \& Chen, G. Effects of surface chemistry on thermal conductance at aluminum-diamond interfaces. Appl. Phys. Lett. 97, 083102 (2010).

25. Liang, Q., Yao, X., Wang, W., Liu, Y. \& Wong, C. P. A three-dimensional vertically aligned functionalized multilayer graphene architecture: an approach for graphene-based thermal interfacial materials. ACS Nano 5, 2392-2401 (2011).

26. Ni, Y. et al. Highly efficient thermal glue for carbon nanotubes based on azide polymers. Appl. Phys. Lett. 100, 193118 (2012).

27. Harikrishna, H., Ducker, W. A. \& Huxtable, S. T. The influence of interface bonding on thermal transport through solid-liquid interfaces. Appl. Phys. Lett. 102, 251606 (2013).
28. Liang, Z., Evans, W., Desai, T. \& Keblinski, P. Improvement of heat transfer efficiency at solid-gas interfaces by self-assembled monolayers. Appl. Phys. Lett. 102, 061907 (2013).

29. OBrien, P. J. et al. Bonding-induced thermal conductance enhancement at inorganic heterointerfaces using nanomolecular monolayers. Nat. Mater. 12, 118 (2013).

30. Taphouse, J. H., Smith, O. N. L., Marder, S. R. \& Cola, B. A. A pyrenylpropyl phosphonic acid surface modifier for mitigating the thermal resistance of carbon nanotube contacts. Adv. Funct. Mater. 24, 465 (2014).

31. Sun, F. et al. Molecular bridge enables anomalous enhancement in thermal transport across hard-soft material interfaces. Adv. Mater. 26, 6093 (2014).

32. Chien, S. K. \& Yang, Y. T. Influence of hydrogen functionalization on therma conductivity of graphene: Nonequilibrium molecular dynamics simulations. Appl. Phys. Lett. 98, 033107 (2011).

33. Kim, J. Y., Lee, J.-H. \& Grossman, J. C. Thermal transport in functionalized graphene. ACS Nano 6, 9050-9057 (2012).

34. Xin, G. et al. Large-area freestanding graphene paper for superior thermal management. Adv. Mater. 26, 4521-4526 (2014).

35. Pasternack, R. M., Amy, S. R. \& Chabal, Y. J. Langmuir 24, 12963-12971 (2008).

36. Chen, G. \& Hui, P. Pulsed photothermal modeling of composite samples based on transmission-line theory of heat conduction. Thin Solid Films 339, 58-67 (1999).

37. Zhao, Y. et al. Pulsed photothermal reflectance measurement of the thermal conductivity of sputtered aluminum nitride thin films. J. Appl. Phys. 96, 4563 (2004).

38. Meier, T. et al. Length-dependent thermal transport along molecular chains. Phys. Rev. Lett. 113, 060801 (2014).

39. Yang, J. et al. Phonon transport through point contacts between graphitic nanomaterials. Phys. Rev. Lett. 112, 205901 (2014).

40. Segal, D., Nitzan, A. \& Hänggi, P. Thermal conductance through molecular wires. J. Chem. Phys. 119, 6840 (2003).

41. Hu, L. et al. Phonon interference at self-assembled monolayer interfaces: Molecular dynamics simulations. Phys. Rev. B 81, 235427 (2010).

42. Markussen, T. Phonon interference effects in molecular junctions. J. Chem. Phys. 139, 244101 (2013).

43. Han, H. et al. Phonon interference and thermal conductance reduction in atomic-scale metamaterials. Phys. Rev. B 89, 180301(R) (2014).

44. Han, H., Li, B., Volz, S. \& Kosevich, Y. A. Ultracompact interference phonon nanocapacitor for storage and lasing of coherent terahertz lattice waves. Phys. Rev. Lett. 114, 145501 (2015).

45. Ni, Y., Chalopin, Y. \& Volz, S. Significant thickness dependence of the thermal resistance between few-layer graphenes. Appl. Phys. Lett. 103, 061906 (2013).

46. Lusk, M. T. \& Carr, L. D. Nanoengineering defect structures on graphene. Phys Rev. Lett. 100, 175503 (2008).

47. Jiang, J.-W., Wang, B.-S. \& Wang, J.-S. First principle study of the thermal conductance in graphene nanoribbon with vacancy and substitutional silicon defects. Appl. Phys. Lett. 98, 113114 (2011).

48. Hughbanks, T. \& Hoffmann, R. Chains of trans-edge-sharing molybdenum octahedra: metal-metal bonding in extended systems. J. Am. Chem. Soc 105, 3528 (1983).

49. Ziman, J. M. Electrons and Phonons (Oxford Univ. Press, 1960).

50. Trew, R. J., Green, D. S. \& Shealy, J. B. AlGaN/GaN HFET reliability. IEEE Microw. Mag. 10, 116127 (2009).

51. Yang, B., Wang, P. \& Bar-Cohen, A. 'Mini-contact enhanced thermoelectric cooling of hot spots in high power devices'. IEEE Trans. Compon. Packag. Technol. 30, 432-438 (2007).

52. Nath, P. \& Chopra, K. L. Thermal conductivity of copper films. Thin Solid Films 20, 5362 (1974).

53. Langer, G., Hartmann, J. \& Reichling, P. Thermal conductivity of thin metal films measured by photothermal profile analysis. Rev. Sci. Instrum 68, 15101513 (1997).

54. Kumar, P. et al. Large-area reduced graphene oxide thin film with excellent thermal conductivity and electromagnetic interference shielding effectiveness. Carbon 94, 494-500 (2015).

55. Hummers, W. S. \& Offeman, R. E. Preparation of graphitic oxide. J. Am. Chem. Soc. 80, 1339-1339 (1958).

56. Plimpton, S. Fast parallel algorithms for short-range molecular dynamics. J. Comput. Phys. 117, 1 (1995).

57. Stuart, S. J., Tutein, A. B. \& Harrison, J. A. A reactive potential for hydrocarbons with intermolecular interactions. J. Chem. Phys. 112, 6472 (2000).

58. Chenoweth, K., van Duin, A. C. \& Goddard, W. A. ReaxFF reactive force field for molecular dynamics simulations of hydrocarbon oxidation. J. Phys. Chem. A 112, 1040-1053 (2008).

59. Sadeghi, H., Sangtarash, S. \& Lambert, C. Enhancing the thermoelectric figure of merit in engineered graphene nanoribbons. Beilstein J. Nanotechnol. 6, 1176 (2015). 


\section{Acknowledgements}

The work is sponsored by EU FP7 programme 'Nanotherm' under the Grant Agreement No 318117. This work is also supported by the National Chinese Science Foundation Projects (51272153 and 61574088) and the Science and Technology Commission of Shanghai Municipality programme (12JC1403900), as well as Shanghai Education Commission programme (Shanghai University Peak Discipline Construction Project), by the Swedish Foundation for Strategic Research (SSF) Frame Project Contract No: SE130061 and SSF Project Contract No: EM11-0002. This work is supported by EPSRC project EP/N017188/1 and by the EU ITN MOLESCO project number 606728 . We acknowledge the financial support from The Production Area of Advance programme, Chalmers University of Technology, Sweden.

\section{Author contributions}

S.V. and J.L. proposed and designed the project; H.H., Y.Z. and N.W. contributed equally to this work; H.H. performed phonon and electron Green's function calculations and molecular dynamics simulations; Y.Z. characterized the samples with Raman spectroscopy, Fourier transform infrared spectroscopy and RTD measurement; N.W. and M.M. prepared GBF and functional materials; M.K.S. conducted the PPR measurement with assistance from Y.Z.; M.E. and H.H. performed FE simulations; Y.F. designed the experiments and analysed the experimental data; L.Y. supervised the experiments on functional materials; H.H., Y.N., K.S., S.Y., Y.A.K. and S.V. analysed and interpreted the phonon transport calculations; H.H. and Z.Y.M. conducted first-principle and Green's function calculations with assistance from S.X., H.S., S.B. and C.L.; H.H. integrated results among different research units and wrote the manuscript.

\section{Additional information}

Supplementary Information accompanies this paper at http://www.nature.com/ naturecommunications

Competing financial interests: The authors declare no competing financial interests.

Reprints and permission information is available online at http://npg.nature.com/ reprintsandpermissions/

How to cite this article: Han, H. et al. Functionalization mediates heat transport in graphene nanoflakes. Nat. Commun. 7:11281 doi: 10.1038/ncomms11281 (2016).

\section{(c) (i)}

This work is licensed under a Creative Commons Attribution 4.0 International License. The images or other third party material in this article are included in the article's Creative Commons license, unless indicated otherwise in the credit line; if the material is not included under the Creative Commons license, users will need to obtain permission from the license holder to reproduce the material. To view a copy of this license, visit http://creativecommons.org/licenses/by/4.0/ 